\title{
Transport modelling for ergodic configurations
}

\author{
A. Runov, N. McTaggart, S. Kasilov ${ }^{1}$, \\ R. Schneider, X. Bonnin, D. Reiter ${ }^{2}$ \\ Max-Planck-Institut für Plasmaphysik, Teilinstitut Greifswald, EURATOM \\ Association, Wendelsteinstraße 1, D-17491 Greifswald, Germany \\ (1) Institute of Plasmaphysics, NSC Kharkov Institute of Physics and Technology, \\ 61108 Kharkov, Ukraine \\ (2) Institut für Plasmaphysik, Forschungszentrum Jülich GmbH, EURATOM \\ Association, Trilateral Euregio Cluster, D-52425 Jülich, Germany
}

\section{Introduction.}

Transport in a magnetized plasma is characterized by strong anisotropy, i.e. the parallel and perpendicular heat conduction coefficients differ by several orders of magnitude. This presents a problem to any numerical method applied to transport equations in real space coordinates. To overcome this problem, a Multiple Coordinate System Approach(MCSA) has been proposed in Ref.[1]. Basically, we choose a coordinate system in which the magnetic field has only one non-zero component. We thus guarantee the separation of the parallel and perpendicular fluxes. If, in our coordinate system $h^{1}=h^{2}=0$, then we have a contribution from $D_{\|}$in $D^{33}$ only such that:

$$
D^{i j}=D_{\perp} g^{i j}+\left(D_{\|}-D_{\perp}\right)\left(h^{3}\right)^{2} \delta_{3}^{i} \delta_{3}^{j}
$$

where $\delta_{j}^{i}$ is a Kronecker symbol, $D_{\|}$and $D_{\perp}$ are (known) transport coefficients along and across magnetic field respectively, $g^{i j}=\left(\nabla x^{i}\right) \cdot\left(\nabla x^{j}\right)$, and $h^{i}=\mathbf{h} \cdot \nabla x^{i}$ are contravariant components of the metric tensor, and of the unit vector along the magnetic field respectively. A general class of such magnetic coordinate systems based on Clebsch stream functions has been proposed in Ref.[1]. To begin, we choose a surface (reference cut) which intersects all field lines of interest and then draw some reasonable (e.g. Cartesian) mesh on this cut. We then trace field lines through the mesh lines. The surfaces we obtain are our coordinate surfaces. The metric of this system can be obtained by field line tracing. Generally we are forced to use several local magnetic coordinate systems because the applicability of a single coordinate system is limited by some toroidal distance (the theoretical limit is the Kolmogorov length). The price for such flexibility is an aperiodic system, i.e. the coordinate surfaces of neighbouring systems overlap arbitrarily at the interface between the two systems. If we try to solve the problem by interpolation, we induce numerical diffusion of the same nature as mentioned before: a contribution of the parallel flux to the perpendicular fluxes. Indeed, if one were to pass a beam (a $\delta$-function) through such a system, one would obtain a response in all four corners of the cell at the interface, and then on the next interface it 
would spread further. The idea realized in Ref.[1] is to use a Monte-Carlo method with an appropriate mapping technique. In this case, the problem becomes one of passing a particle from one coordinate system to another, instead of mesh re-interpolation. Here, we present the latest modifications of the MCSA algorithm allowing for long parallel steps. We also present a finite-difference realization of the MCSA in which we minimize the numerical diffusion induced by interpolation on the interface by optimizing the mesh on the reference cut, i.e. we generate the mesh by field line tracing itself.

\section{Monte-Carlo method.}

This approach is straightforward: we model a diffusion-like equation using the random walk of Brownian particles. Sooner or later, particles must travel to the neighbouring coordinate system, i.e. their perpendicular coordinates must be transformed with high accuracy. It is easy to see that this problem reduces to a $2 \mathrm{D}$ transformation (mapping) between the reference cuts. It is solved by means of an interpolated cell mapping procedure, as described in [1]. For stellarator applications the coordinates should be optimized. Indeed, the reference cut mesh does not have to be Cartesian - it is more natural to choose the magnetic surfaces where ever they exist (and some interpolation where there are no surfaces), use the surface label as a measure of radial distance, and the VMEC angle-like variable $\theta$ as the second family of curves, as described in [2]. The restriction on the time step in Monte Carlo is clear: a single particle step must be small compared to the gradient length of the diffusion coefficient. Here, the parallel diffusion coefficient depends on both plasma and magnetic field parameters. The plasma parameter dependence comes from the non-linear parallel heat conduction, whereas the magnetic field dependence comes from $\left(h^{3}\right)^{2}$ and the metric determinant:

$$
\begin{aligned}
D_{\perp}^{i j} & =D_{\perp e}\left[g^{i j}-\left(h^{3}\right)^{2} \delta_{3}^{i} \delta_{3}^{j}\right], \quad D_{\|}^{33}=D_{\| e}\left(h^{3}\right)^{2}, \\
V_{\perp c}^{i} & =\mathbf{V}_{\perp e} \cdot \nabla x^{i}+\frac{1}{\sqrt{g}} \frac{\partial}{\partial x^{j}} \sqrt{g} D_{\perp}^{i j}, \quad V_{\| c}^{3}=V_{\| e} h^{3}+\frac{1}{\sqrt{g}} \frac{\partial}{\partial x^{3}} \sqrt{g} D_{\|}^{33} .
\end{aligned}
$$

The problem arises near the inner boundary of the domain. Here there is a high constant temperature resulting in very large parallel heat conduction, while the characteristic length of the magnetic field is one period or even the distance between the coils. This means that reproducing this constant numerically will be very CPU intensive. To allow for long parallel steps, magnetic field dependencies must be eliminated. First, the 3rd coordinate is modified according to

$$
x_{(m)}^{3}=S_{(m)} \int_{\varphi_{m i n}^{(m)}}^{\varphi} \mathrm{d} \varphi^{\prime} \frac{(B)^{2}}{B^{\varphi}}+\varphi_{m i n}^{(m)}, \quad S_{(m)}=\left(\varphi_{\max }^{(m)}-\varphi_{\min }^{(m)}\right)\left(\int_{\varphi_{\min }^{(m)}}^{\varphi_{\max }^{(m)}} \mathrm{d} \varphi^{\prime} \frac{(B)^{2}}{B^{\varphi}}\right)^{-1},
$$

(integration is along field line). The surfaces $x^{3}=$ const become something like potato chips instead of planes to keep the combination $\left(h^{3}\right)^{2} \sqrt{g}$ constant along the field line and therefore to eliminate its derivative from the convection term.

Second, the weights of test particles are manipulated to keep the coefficient $D^{33}$ 
itself "almost constant" (it would be exactly constant for constant temperature):

$$
w=w_{0} \lambda \sqrt{g}, \quad \text { where } \quad \lambda\left(x^{1}, x^{2}\right)=\left(\frac{\partial(R, Z)}{\partial\left(x^{1}, x^{2}\right)}\right)^{-1} \frac{S_{(m)}}{\hat{B}_{\varphi}} .
$$

The resulting efficiency of the code is about two orders of magnitude better. The results for limiter-like configuration for W7-X are presented in Fig.1. The electron temperature in the island is almost constant, the ion temperature has a noticeable gradient across the island and the density shows an even stronger gradient.

\section{Finite-Difference Scheme.}

Here we describe the finite-difference approach using the local magnetic coordinates mentioned above. The idea is to minimize numerical diffusion by means of an optimized mesh. The mesh points are generated at the reference cuts by a field line tracing code. This idea is straightforward for the open field lines, they simply start and end at the wall. Any other field line can be treated as "almost closed" as long as the following criterion is fulfilled:

$$
\Delta \ll L_{\|} \sqrt{\chi_{\perp} / \chi_{\|}}
$$

where $\Delta$ is the excursion between the start and end points on the cut, and $L_{\|} \approx 2 \pi R N$ is the distance between the same points measured along the field line. In W7X $R \approx$ $5.5 \mathrm{~m}$ and we choose $N \approx 100$ toroidal turns. We select only those field lines which come within $\Delta=1 \mathrm{~mm}$ of closing upon themselves. Using these values for $\Delta, R$ and $N$ for building a mesh, the resulting induced numerical diffusion is of order $10^{-4} \mathrm{~m}^{2} / \mathrm{s}$, i.e. negligible compared to the physical $\chi_{\perp}$. The particular set of magnetic coordinates for the FDS is constructed in cylindrical coordinates $(R, Z, \Phi)$ with $\Phi$ being the toroidal angle. First, we introduce a set of equally spaced toroidal (reference) cuts with $\Delta \Phi=2 \pi / M$. The number of toroidal cuts (usually $M=20$ ) must be such that the distance between neighboring cuts is much smaller than the Kolmogorov length. This means that field line segments connecting three neighbouring cuts are not long enough to exhibit ergodicity. The penalty one pays for this method is an unstructured mesh. In finite difference modeling, we must determine the neighbors for each point in order to solve a discretized transport equation across the plot. Each point has, on average, 6 radial neighbours on the $2 \mathrm{D}$ cut. To find the neighbors, we use the Delaunay triangulation method and we apply constraints to preserve the structural information about magnetic surfaces where ever they exist. These constraints add a lot of complexity and it is necessary to analyze the grid after triangulation, identify the problem regions and correct them. The first results for W7X are presented in Fig.2. which shows a Poincaré plot, the triangulation, and the final temperature solution.

\section{References}

[1] A.M.Runov, et al., Physics of Plasmas, vol.8, No.3 (2001) 916

[2] A.M.Runov, et al., J. Nucl. Materials, vol.313-316, (2003) 1292 


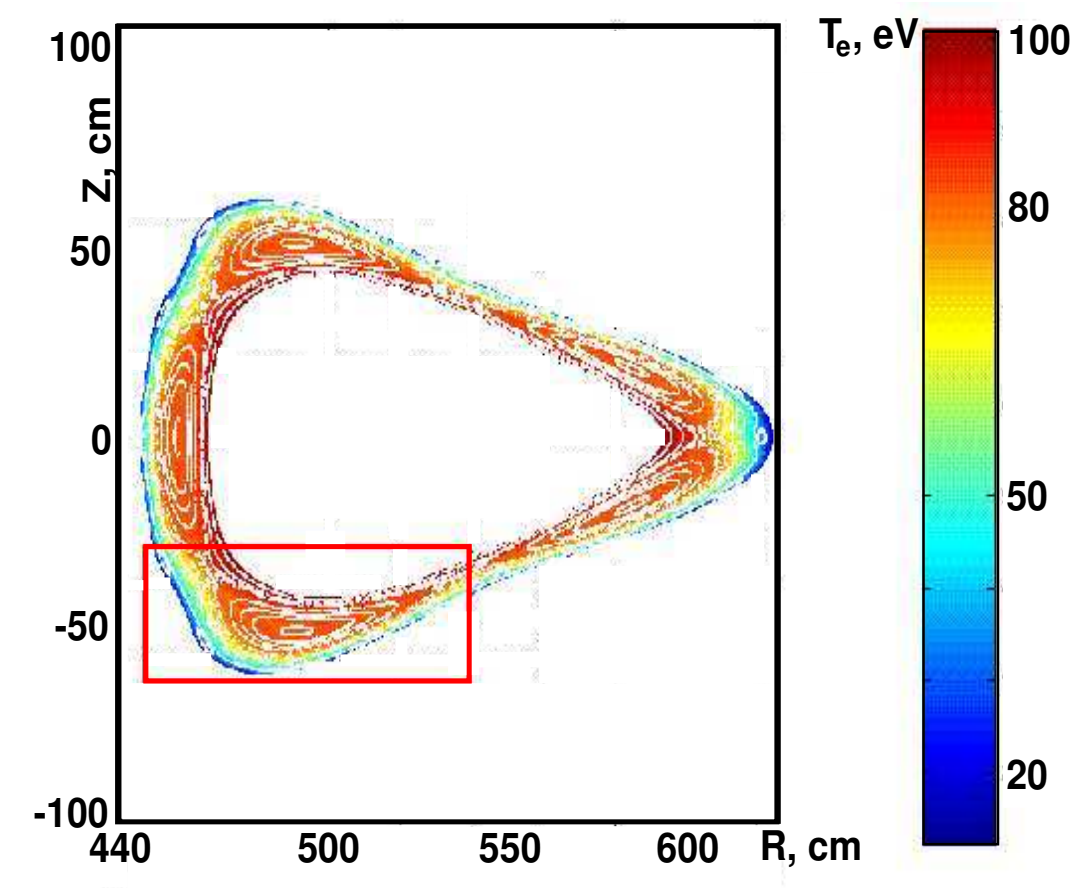

00

\section{0}

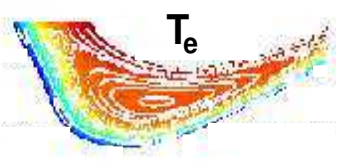

50
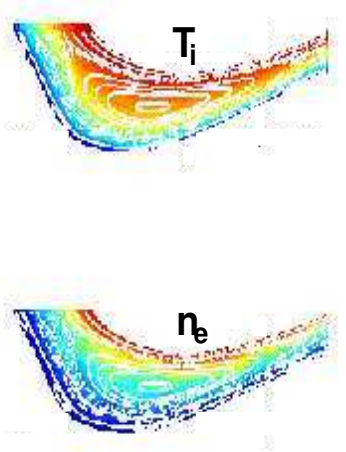

Figure 1: Monte-Carlo calculations for W7X geometry.
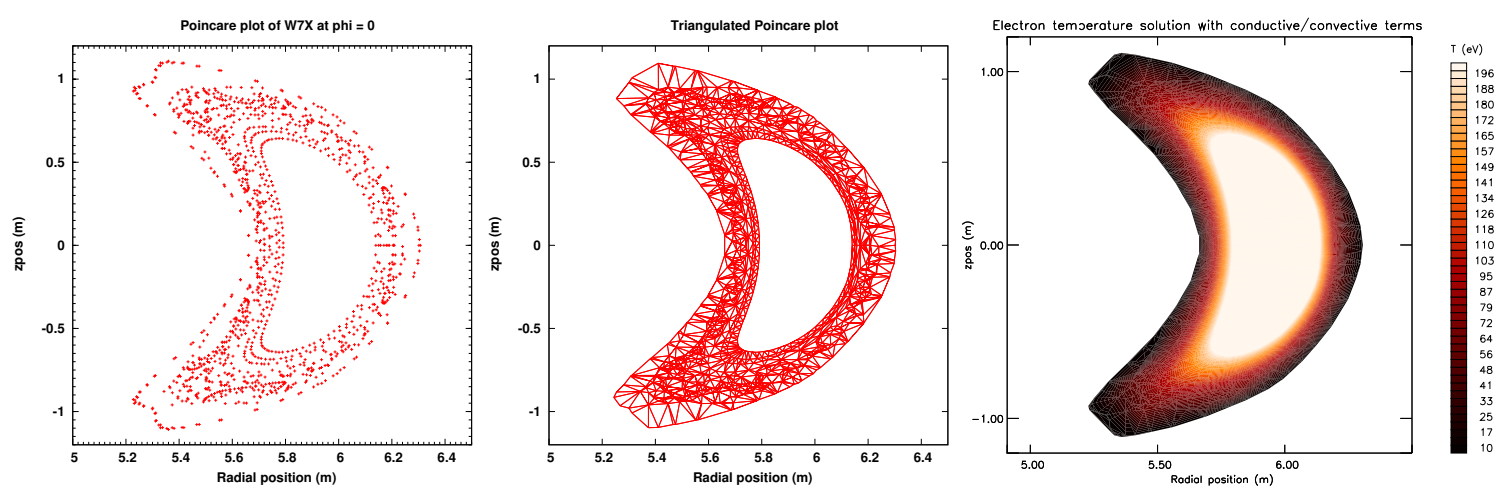

Figure 2: Poincaré plot, triangulation and electron temperature contours for toroidal section with $\Phi=0$. 